\title{
Control glicémico del paciente quirúrgico
}

\author{
Glycemic control of the surgical patient \\ Controle glicêmico do paciente cirúrgico
}

Lorena Bruno ORCID: 0000-0001-5678-7971 Internista. Diabetólogo. Ex -Asistente

Clínica Médica. UDA Diabetes.

Mariela Garau

ORCID: 0000-0002-9632-7539

Profesora Agregada Departamento de Métodos Cuantitativos. Facultad Medicina.

Lorena Barreneche

ORCID: 0000-0003-2060-500X Postgrado Medicina Interna y Diabetología.

Laura Ureta

ORCID: 0000-0001-5388-5437

Postgrado Medicina Interna.

Santiago Algorta

ORCID: 0000-0002-7613-3809

Postgrado Medicina Interna

Roberto Di Lorenzi ORCID: 0000-0003-1268-940X Internista. Diabetólogo. Ex -Asistente Clínica Médica. UDA Diabetes.

María Eugenia Ruíz Díaz ORCID: 0000-0003-3297-2739 Internista. Diabetólogo. UDA

Diabetes.

Iris Melone

ORCID: 0000-0003-0431-4173 Licenciada Psicología. UDA Diabetes.
Resumen: Introducción. La diabetes mellitus es una patología de alta prevalencia, con mayor morbimortalidad en el paciente quirúrgico, tanto por la hipo como la hiperglicemia. Objetivos. Determinar si el control metabólico glicémico cumple las recomendaciones internacionales. Metodología. Se realizó un estudio descriptivo de todos los pacientes internados en áreas quirúrgicas. Se analizaron todas las glicemias capilares registradas en la historia clínica. Resultados. Se captaron 34 pacientes (predomina sexo masculino y con diabetes mellitus tipo 2), 21 fueron sometidos a una intervención quirúrgica, la mortalidad fue del 6\%. La glicemia promedio fue superior a la recomendada internacionalmente, constituye episodios de hiperglicemia. Asimismo se detectó la presencia de hipoglicemia. Conclusiones. Es deseable establecer estrategias para optimizar el control metabólico en la población con diabetes mellitus internada en áreas quirúrgicas.

Palabras claves: diabetes mellitus, hipoglicemia, hiperglicemia, cirugía.

Abstract: Introduction. Diabetes mellitus is a highly prevalent pathology, with higher morbidity and mortality in the surgical patient, both due to hypo and hyperglycemia. Goals. Determine if glycemic metabolic control meets international recommendations. Methodology. A descriptive study of all patients admitted to surgical areas was carried out. All capillary blood glucose levels recorded in the clinical history were analyzed. Results. 34 patients were captured (predominantly male and with type 2 diabetes mellitus), 21 underwent surgical intervention, mortality was $6 \%$. The average glycemia was higher than the internationally recommended, it constitutes episodes of hyperglycemia. Likewise, the presence of hypoglycemia was detected. Conclusions. It is desirable to establish strategies to optimize metabolic control in the population with diabetes mellitus admitted to surgical areas.

Key words: diabetes mellitus, hypoglycemia, hyperglycemia, surgery.

Resumo: Introdução. O diabetes mellitus é uma patologia de alta prevalência, com maior morbimortalidade no paciente cirúrgico, tanto por hipo quanto por hiperglicemia. Metas. Determine se o controle metabólico glicêmico atende às recomendações internacionais. Metodologia. Foi realizado um estudo descritivo de todos os pacientes admitidos em áreas cirúrgicas. Todos os níveis de glicose no sangue capilar registrados na história clínica foram analisados. Resultados. 34 pacientes foram capturados (predominantemente do sexo masculino e com diabetes mellitus tipo 2), 21 foram submetidos a intervenção cirúrgica, a mortalidade foi de $6 \%$. A média da glicemia foi superior ao recomendado internacionalmente, constituindo episódios de hiperglicemia. Da mesma forma, foi detectada a presença de hipoglicemia. Conclusões. É desejável estabelecer estratégias para otimizar o controle metabólico na população com diabetes mellitus admitida em áreas cirúrgicas.

Palavras chave: diabetes mellitus, hipoglicemia, hiperglicemia, cirurgia.

Recibido: 06/10/2019 Aceptado: 12/09/2020

Unidad Docente Asistencial - Atención integral del paciente diabético. Hospital Pasteur. Administración Servicios del Estado (ASSE). Facultad de Medicina. Universidad de la República. UdelaR. Uruguay.

Correspondencia: E-mail: brunogonlor76@gmail.com 


\section{Introducción}

La Diabetes Mellitus (DM) es una patología de alta prevalencia a nivel mundial, que afecta actualmente 425 millones de personas (8.8\%), estimándose para 2045 un aumento a 693 millones de personas (9.9\%), según International Diabetes Federation (IDF). ${ }^{(1)}$ Nuestro país reporta una prevalencia de $8 \%$ en población general. ${ }^{(2)}$

Estas cifras aumentan drásticamente si se considera la población hospitalizada, donde a nivel mundial la prevalencia de DM oscila entre $16-20 \%{ }^{(3-5)}$; en Uruguay contamos con cifras entre 22 - 27\% de DM para la hospitalización en el subsector asistencial público ${ }^{(6,7)}$ y de $13 \%$ en el subsector privado. ${ }^{(8)}$

El paciente con DM tiene mayor probabilidad de ser operado, registrandoestadías hospitalarias más prolongadas y mayor morbi -mortalidad que el paciente sin DM. Esto se relaciona con múltiples factores, que incluyen: la presencia tanto de hipo como de hiperglicemia (7,9), la comorbilidad propia de las repercusiones micro y macroangiopáticas y el efecto de la cirugía sobre la glicemia. ${ }^{(3,9-12)}$

La hiperglicemia durante la internación se relaciona con complicaciones, tanto infecciosas ${ }^{(7,9)}$ (efecto pro-inflamatorio de la hiperglicemia, asociado a alteración de la función leucocitaria) como cardiovasculares (hipertrombicidad por alteraciones de la función plaquetaria asociada a complicaciones macrovasculares y arrítmicas). ${ }^{(3,9-15)}$

La hipoglicemia también aumenta la morbi-mortalidad, ya que aumenta la tasa de infección intrahospitalaria, de cardiopatía isquémica, insuficiencia cardiaca y ataque cerebrovascular; la mortalidad relacionada a hipoglicemia se ve aumentada hasta un año después del alta. ${ }^{(16-17)}$

Las repercusiones de la DM más relevantes en relación al peri-operatorio son: neuropatía autonómica, nefropatía, cardiopatía isquémica y enfermedad cerebrovascular. ${ }^{(4,18)}$

La cirugía y la anestesia general producen una respuesta de estrés neuroendócrina con liberación de hormonas contrarreguladoras, que contribuyen a producir hiperglicemia (resistencia a la insulina, disminución de la utilización periférica de insulina, alteración de la secreción de insulina), los que sumados al ayuno quirúrgico, conllevan a un aumento de la lipólisis y del catabolismo proteico) pudiendo provocar descompensaciones hiperglicémicas graves como cetoacidosis diabética y estado hiperosmolar no cetósico, generando mayor morbimortalidad. ${ }^{(4,18)}$

El peri-operatorio es una situación clínica compleja, donde el médico clínico debe intentar mantener la homeostasis glicémica en un paciente sin secreción insulínica (DM tipo 1) o con alteración en la secreción de la misma asociado a grados variables de insulinorresistencia (DM tipo 2), sometido a stress metabólico y en el cual la vía oral se encuentra suspendida en forma frecuente.

Según recomendaciones internacionales,,$^{(9,10,12,18,19)}$ para el peri-operatorio se desaconsejan cifras de glicemia capilar $<70 \mathrm{mg} / \mathrm{dl}$ (hipoglicemia) y $>180 \mathrm{mg} / \mathrm{dl}$ (hiperglicemia) en cualquier momento del día.

Entre 70 y $180 \mathrm{mg} / \mathrm{dl}$ se establece una sub categoría de 110-180 mg/dl como la más recomendable y segura para la mayoría de los pacientes, independiente del momento de realizada la determinación.

Con el objetivo de conocer si el comportamiento metabólico de los pacientes ingresados en área quirúrgico se ajusta a estas pautas internacionales, es que se realiza el presente trabajo.

\section{Metodología}

Se realizó un estudio descriptivo, de todos los pacientes diabéticos que ingresaron en un lapso arbitrario de 20 días (mes setiembre 2019) en áreas quirúrgicas (cirugía general, vascular, plástica, urología, otorrinolaringología) en un hospital general de adultos de tercer nivel de atención (Hospital Pasteur, Montevideo-Uruguay), realizando el seguimiento individual de cada uno hasta su egreso. La inclusión se realizó independiente de si requirieron cirugía de urgencia, coordinación o si incluso no fueron sometidos a la misma. Se excluyeron del estudio los pacientes egresados de unidad cuidados intensivos.

Se obtuvieron datos de la historia clínica (edad, sexo, tipo de DM, tiempo de evolución de la misma, causa de ingreso, mortalidad). 
De cada paciente, se analizaron todas las glicemias capilares que figuraban en los registros de enfermería, independientemente de la relación con la ingesta o si presentaban vía oral suspendida. Se incluyeron los registros de glicemia capilar de la ficha anestésica para los pacientes que requirieron cirugía.

Para el control de la glicemia se utilizó en todos los casos un glucómetro Nipro True Results.

Análisis estadístico: La normalidad de la distribución se investigó con test de Shapiro Wilk. Las variables cuantitativas se compararon mediante test de T o test de U de Man Whitney según presentaran distribución normal o no. Se consideró significativo $\mathrm{p}<0.05$. Se utilizó SPSS v.15

\section{Resultados}

Se captaron 34 pacientes diabéticos, correspondientes al $24 \%$ de los internados en el período de estudio. En la tabla 1 se presentan los motivos de ingreso.

La edad promedio de los pacientes fue de $60.8 \pm 14.1$ años, el $74 \%$ eran de sexo masculino, el $85 \%$ tenía DM tipo 2 y el $15 \%$ restante DM tipo 1, el tiempo promedio de evolución de la diabetes fue de $11.8 \pm 9.0$ años.

21/34 pacientes (62\%) fue sometida a cirugía. La mortalidad global fue de 2/34 (6\%).

Tabla 1: Motivos de ingreso de pacientes diabéticos internados en áreas quirúrgicas

\begin{tabular}{|l|l|}
\hline Causa de ingreso & $\begin{array}{l}\text { Frecuencia Absoluta } \\
(\mathbf{N}=\mathbf{3 4 )}\end{array}$ \\
\hline Infección Pie diabético & 12 \\
Abscesos (perihepático, renal, empiema) & 3 \\
Celulitis de muñón & 2 \\
Osteomielitis & 1 \\
Infección de úlcera de mmii & 1 \\
Infección de abordaje vascular reciente & 1 \\
\hline Tumor Urológico & 5 \\
Tumor Digestivo & 1 \\
\hline Patología vascular & 4 \\
\hline Otros & 4 \\
\hline
\end{tabular}

Se analizaron los promedios de glicemia capilar de cada paciente. En la gráfica 1 se presenta la distribución de la glicemia promedio según sexo

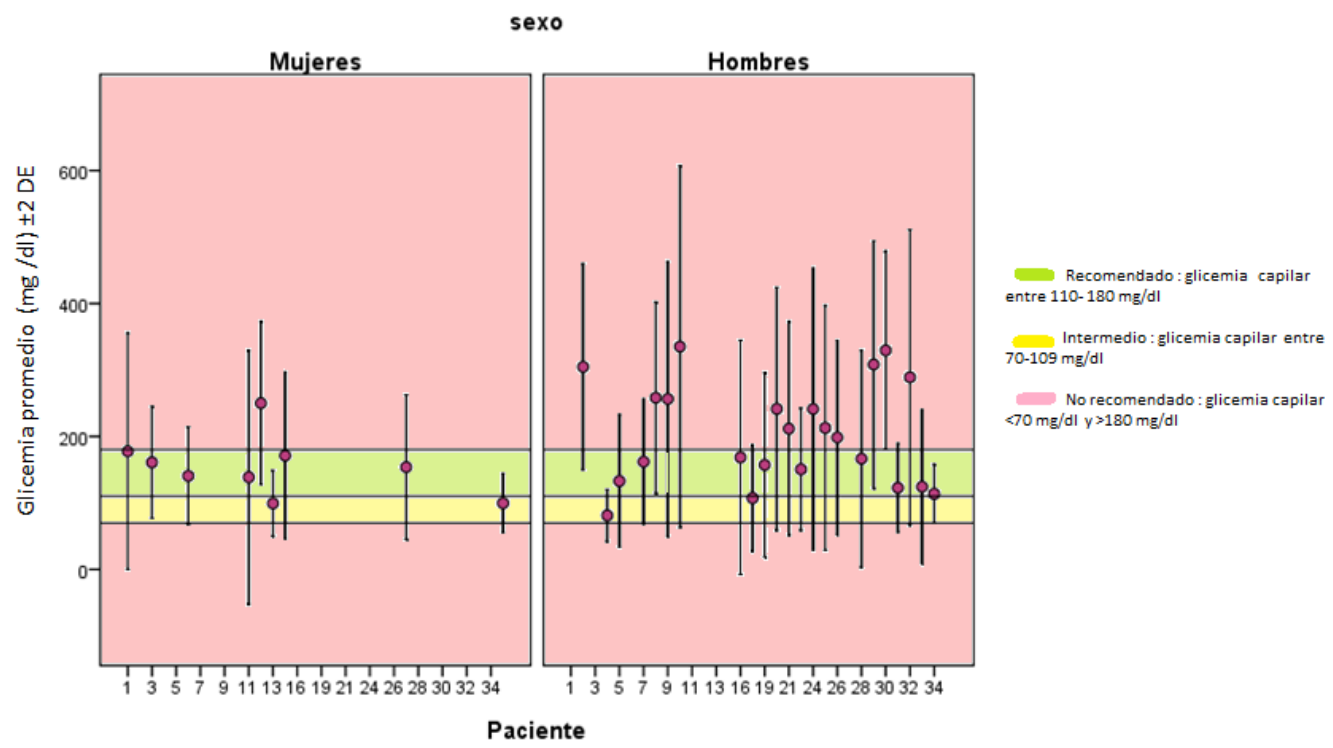

La media global fue $189.2 \mathrm{mg} / \mathrm{dl}$ (diferenciado por sexo - hombres: media de glicemia capilar $201 \mathrm{mg} / \mathrm{dl}$, mujeres: media de glicemia capilar $154 \mathrm{mg} / \mathrm{dl}$ ). 
Diferencia estadísticamente significativa (Test de t con valor $p=0.041$ ).

10/34 pacientes (29\%) presentó al menos un episodio de hipoglicemia (glicemia capilar $<70$ $\mathrm{mg} / \mathrm{dl})$.

\section{Discusión}

Se constató una prevalencia de $24 \%$ de DM en la internación quirúrgica, similar a la reportada en el mismo centro para sala de cirugía $(26.9 \%)^{(7)}$, y superior a la citada por países desarrollados con reportes entre $10-20 \%$ en distintas publicaciones. ${ }^{(3-5)}$

Al igual que series internacionales ${ }^{(12,19)}$, se trata predominantemente de adultos del sexo masculino. Similar a los datos reportados por la serie británica de Jackson ${ }^{(20)}$, son sobre todo DM tipo 2 y de larga evolución de la enfermedad.

La infección fue la causa de ingreso más frecuente, especialmente la patología infecciosa asociada a pie diabético.

Esto marca una diferencia relevante con las series internacionales, donde las principales patologías por las que ingresan los diabéticos varían según el centro pero están representadas por la patología digestiva, genitourinaria y el trauma, seguidos de la patología vascular y donde la patología infecciosa relacionada a pie diabético tiene un registro mínimo. ${ }^{(12,20)}$ La mayoría de los pacientes tuvo sanción quirúrgica.

La glicemia capilar promedio de la serie supera las recomendaciones; los hombres presentaron una glicemia promedio significativamente superior a la de las mujeres. Las causas de este hecho pueden ser multifactoriales y exceden los objetivos del presente estudio. En su publicación Umpierrez ${ }^{(12)}$ encontró una media superior a la recomendada, en una cohorte que incluyó exclusivamente DM tipo 2.

La hipoglicemia registrada del $29 \%$ fue similar a la reportada por Umpierrez ${ }^{(12)}$, e inferior a la citada por Steely ( $37 \%$ en cirugía vascular) ${ }^{(21)}$ y a la aportada por estudios en el paciente crítico (45\%). ${ }^{(22)}$

Como debilidad del estudio se destaca el tiempo acotado de captación de casos; sin embargo, se considera que aporta insumos para conocer la realidad de nuestro medio.

\section{Conclusiones}

Los pacientes con DM son un porcentaje importante de los ingresos a áreas quirúrgicas asistenciales, ingresando en su mayoría por una patología prevenible como es el pie diabético. El promedio de glicemia capilar es superior al recomendado internacionalmente, siendo fundamental implementar estrategias para mejorar esta realidad.

\section{Bibliografía}

1- International Diabetes Federation. IDF Diabetes Atlas update poster, 8th ed. Brussels: IDF, 2017.

2- Ferrero Petean RT, Gracía MV. Encuesta de prevalencia de la diabetes en el Uruguay. Arch. Med. Int (Montevideo). 2005; 26; 1: 07-12.

3- Frisch A , Chandra P , Smiley D , Peng L , Rizzo M , Gatcliffe C , et al. 2010 Prevalence and clinical outcome of hyperglycemia in the perioperative period in noncardiac surgery. Diabetes Care 33:17831788.

4- Levy N, Penfold N, Mythen M. Perioperative management of diabetes and the emerging role of anaesthetists as perioperative physicians. Br J Anaesth. 2016 Apr; 116(4):443-7. doi: 10.1093/bja/ aew049.

5- National Health Service Diabetes. National diabetes inpatient audit 2010 full report [Internet]. NHS: Wales, 9 May 2019 [cited mar 31 2019]. Available in: https://digital.nhs.uk/data-and-information/ clinical-audits-and-registries/national-diabetes-inpatient-audit

6- Bruno L, Di Lorenzi R, Pandolfi M, Aseretto M, Javiel G. Control glucémico en paciente internado. Rev Urug Med Int. [Internet]. 2017 Ago [citado 2019 Mar 31]; 2(2): 22-28.

7- Ibarra A. Prevalencia y características clínicas de pacientes diabéticos ingresados en un hospital general. Arch. Med Int (Montevideo). 2015 Jul; 37(2): 57-60. 
8- Serra MP, Chichet A, Fernández ML, Vadell ML, Castrillón C, Giachero V, et al. Prevalencia de diabetes en pacientes internados: factores socioeconómicos-culturales; educación de la enfermedad y dificultades para el tratamiento. Rev. Méd. Urug. 2003; 19: 34-44.

9- Barker P, Creasey PE, Dhatariya K, Levy N, Lipp A, Nathanson MH, et al. Perioperative management of the surgical patient with diabetes 2015. Anaesthesia. 2015; 70: 1427-1440.

10- Aldam P, Levy N, Hall GM. Perioperative management of diabetic patients: new controversies. $\mathrm{Br} \mathrm{J}$ Anaesth 2014; 113: 906-9.

11- Clement S, Braithwaite SS, Magee MF, Ahmann A, Smith EP, Schafer RG, et al. Management of diabetes and hyperglycemia in hospitals. Diabetes Care.2004; 27:553-597.

12- Umpierrez GE , Smiley D , Jacobs S , Peng L , Temponi A , Mulligan P , et al. Randomized study of basal bolus insulin therapy in the inpatient management of patients with type 2 diabetes undergoing general surgery (rabbit surgery). Diabetes Care. 2011; 34:256-261.

13- Hattori Y, Hattori S, Sato N, Kasai K. High-glucose-induced nuclear factor kappaB activation in vascular smooth muscle cells. Cardiovasc Res. 2000; 46:188- 197.

14- Mowat A, Baum J. Chemotaxis of polymorphonuclear leukocytes from patients with diabetes mellitus. N Engl J Med. 1971; 284:621-627.

15- Pandolfi A, Giaccari A, Cilli C, Alberta M, Morviducci L, Filippis ED, et al. Acute hyperglycemia and acute hyperinsulinemia decrease plasma fibrinolytic activity and increase plaminogen activator inhibitor type 1 in the rat. Acta Diabetol. 2001 38:71-77.

16- Turchin A, Matheny ME, Shubina M, Scanlon JV, Greenwood B, Pendergrass ML. Hypoglycemia and clinical outcomes in patients with diabetes hospitalized in the general ward. Diabetes Care. 2009 Jul;32(7):1153-7. doi: 10.2337/dc08-2127.

17- Kagansky N, Levy S, Rimon E, Cojocaru L, Fridman A, Ozer Z, et al. Hypoglycemia as a predictor of mortality in hospitalized elderly patients. Arch Intern Med. 2003 Aug 11-25;163(15):1825-9. doi: 10.1001/archinte.163.15.1825.

18- Dhatariya K, Levy N, Kilvert A, Watson B, Cousins D, Flanagan D, et al; Joint British Diabetes Societies. NHS Diabetes guideline for the perioperative management of the adult patient with diabetes. Diabet Med. 2012 Apr; 29(4):420-33. doi: 10.1111/j.1464-5491.2012.03582.x.

19- Standards of Medical Care in Diabetes: 2019 Diabetes Care. Jan 2019; 42 (Suppl 1) S4-S6.

20- Jackson MJ, Patvardhan C, Wallace F, Martin A, Yusuff H, Briggs G, Malik RA; NWRAG Peri-Op Diabetes Audit Group. Perioperative management of diabetes in elective patients: a region-wide audit. Br J Anaesth. 2016 Apr;116(4):501-6. doi: 10.1093/bja/aev554.

21- Steely AM, Smith L, Callas PW, Nathan MH, Lahiri JE, Stanley AC, et al. Prospective study of postoperative glycemic control with a standardized insulin infusion protocol after infrainguinal bypass and open abdominal aortic aneurysm repair. Ann Vasc Surg. 2017; 44:211-20.

22- NICE-Sugar Study Investigators. Hypoglycemia and risk of death in critically ill patients. N Eng J Med 2012; 367:1108-1118.

\section{Aporte cada autor al trabajo}

Lorena Bruno: Concepción y diseño del trabajo. Recolección de datos. Análisis e interpretación de los datos. Redacción del manuscrito.

Mariela Garau: Análisis e interpretación de datos. Análisis estadístico. Revisión crítica del manuscrito.

Lorena Barreneche: Recolección de datos. Análisis e interpretación de los datos.

Laura Ureta: Recolección de datos. Análisis e interpretación de los datos.

Santiago Algorta: Recolección de datos. Análisis e interpretación de los datos.

Roberto Di Lorenzi: Concepción y diseño del trabajo. Revisión crítica del manuscrito.

Eugenia Ruiz Díaz: Recolección de datos. Redacción del manuscrito.

Iris Melone: Recolección de datos. Redacción del manuscrito. 